\title{
THE COHESIVE PROPERTY
}

\author{
GERALD JUNGCK
}

\begin{abstract}
We introduce the concept of cohesive families of neighborhood bases. We thereby obtain conditions necessary and sufficient to ensure that a separable space be second countable, and sufficiency conditions for complete collectionwise normality. As by-products we obtain metrizability criteria. We prove, e.g., that a $T_{1}$ space is metrizable iff it has a refined development $\left\{G_{n}: n \in N\right\}$ such that $\left\{B_{p}\right.$ : $p \in X\}$ with $B_{p}=\left\{\operatorname{St}\left(p, G_{n}\right): n \in N\right\}$ is cohesive.
\end{abstract}

1. Preliminaries. A basic result in topology states that any second countable space is separable, whereas the converse is false. The question as to precisely what property must be added to separability to produce second countability appears to remain unanswered. We provide an answer via the concept of "cohesive" families, a concept we define shortly. We then further apply this cohesive property to obtain criteria for collectionwise normality and metrizability, and a characterization of metrizability in terms of uniformly continuous semimetrics. First, however, we comment on terminology and notation.

If $p$ is a point in a topological space $(X, T)$, a neighborhood of $p$ is a set $V(p)$ such that $p \in 0 \subset V(p) \subset X$ for some $0 \in T$. We refer to a neighborhood basis $B_{p}$ at $p$ as a "local base at $p$ " if $B_{p} \subset T$. And $B_{p}$ is "monotone" if $B_{p}$ is linearly ordered by set inclusion, with the usual requirement that $V_{n+1}(p) \subset V_{n}(p)$ for $n \in N$ when $B_{p}$ is countable ( $N$ denotes the set of positive integers). A $T_{1}$ space $(X, T)$ is a semimetric space iff there is a function $d$ on $X \times X$ into the nonnegative reals (called a semimetric) such that $d(x, y)=d(y, x), d(x, y)=0$ iff $x=y$, and $d$ is compatible with the topology $T$ (i.e., if $M \subset X, x \in \bar{M}$ iff $\inf \{d(x, y): y \in M\}=$ $0)$. We let $S(x, r)$ denote the set $\{y \in X: d(x, y)<r\}$. And $(X, T)$ is developable iff there is a sequence $G=\left\{G_{n}: n \in N\right\}$ of open covers of $X$ such that $\left\{\operatorname{St}\left(x, G_{n}\right)\right.$ : $n \in N\}$ is a local base at $x$ for each $x \in X$. $G$ is called a development for $X$.

2. The cohesive concept and applications. Let $(X, T)$ be a space and let $B$ be any basis for $T$. If $B_{p}=\{0 \in B: p \in 0\}$, then the family $\left\{B_{p}: p \in X\right\}$ is a "cohesive" family.

Definition 2.1. Let $(X, T)$ be a topological space, and for each $p \in X$ let $B_{p}$ be a neighborhood basis at $p$. The family $\left\{B_{x}: x \in X\right\}$ is cohesive at $p \in X$ iff the following obtains.

(*) If $0 \in T$ and $p \in 0$, there exists $V \in B_{p}$ such that $x \in V$ implies that $p \in U$ and $U \subset 0$ for at least one $U \in B_{x}$.

Received by the editors August 29, 1980.

1980 Mathematics Subject Classification. Primary 54D15, 54E25, 54E30, 54E35.

Key words and phrases. Cohesive families, semimetric, developable, metrizable. 
We say that the family is cohesive iff it is cohesive at each point of $X$.

As noted above, any space has cohesive families of neighborhood bases. We can sometimes be selective.

EXAMPLE 2.2. If $(X, d)$ is a metric space and $B_{p}=\{S(p, 1 / n): n \in N\}$, then $\left\{B_{p}: p \in X\right\}$ is a cohesive family in which each $B_{p}$ is monotone and countable.

Of course, very nice spaces may have monotone countable local bases which generate noncohesive families.

EXAMPLE 2.3. Let $X=R$, the reals with the usual topology, and $Z$ the set of integers. If $x \in(i, i+1)$ for some $i \in Z$, let $B_{x}=\{(x-1 / n, x+1 / n) \cap(i, i+$ 1): $n \in N\}$, and for $x \in Z$ let $B_{x}=\{(x-1 / n, x+1 / n): n \in N\}$. Then each $B_{x}$ is a local base and the family $\left\{B_{x}: x \in X\right\}$ is cohesive on $X-Z$ but at no point of $Z$.

The next example motivates our first result.

EXAMPLE 2.4. If $X=R$ and $T$ is the lower limit topology generated by the half-open intervals $[a, b)$, then $(X, T)$ is first countable and separable (and paracompact, [6, p. 175]), but not second countable. The following theorem tells us that if $B_{p}$ is a countable local base for each $p$, then the family $\left\{B_{p}: p \in X\right\}$ cannot be cohesive.

THeOREM 2.5. A topological space $(X, T)$ is second countable if and only if it is separable and has a countable local base $B_{p}$ at each $p \in X$ such that the family $\left\{B_{p}\right.$ : $p \in X\}$ is cohesive.

Proof. (Necessity.) Since any second countable space is separable, we need only prove the cohesive property. So let $B$ be a countable base for $T$. For $p \in X$ let $B_{p}=\{V: V \in B$ and $p \in V\}$. Clearly, $B_{p}$ is a countable local base. To see that $\left\{B_{p}: p \in X\right\}$ is cohesive, let $p \in 0 \in T$. Since $B$ is a basis for $T$, there exists $V \in B$ such that $p \in V$ and $V \subset 0$. Now $V \in B_{p}$ by definition of $B_{p}$. But since $V \in B_{x}$ for any $x \in V$, condition (*) in the definition of cohesive families is trivially satisfied with $U=V$.

(Sufficiency.) Let $\left\{B_{p}: p \in X\right\}$ be a cohesive family assured by the hypothesis, and let $D$ be a countable dense subset of $X$. Define $B=\cup\left\{B_{p}: p \in D\right\}$. Clearly, $B$ is countable. We assert that $B$ is a basis for $T$. First note that $B \subset T$. Now let $0 \in T$ and $p \in 0$. Let $V \in B_{p}$ which satisfies property (*) with respect to the given 0 and $p$. Since $D$ is dense and $V$ is open, there exists $x \in D \cap V$. By (*) $p \in U \subset 0$ for some $U \in B_{x}$. But $U \in B_{x}$ and $x \in D$ imply that $U \in B$, and the theorem is proved.

We next consider the cohesive concept in the context of monotone neighborhood bases $B_{p}$, with the reminder that a $T_{1}$ space is collectionwise normal iff every discrete collection of sets can be covered by a pairwise disjoint collection of open sets, each of which covers just one of the original sets.

THEOREM 2.6. Let $(X, T)$ be a $T_{1}$ space which has a neighborhood basis $B_{p}$ at each $p \in X$ such that $B=\left\{B_{p}: p \in X\right\}$ is cohesive. If $X$ has a dense subset $D$ such that $B_{p}$ is monotone for each $p \in D$, then $(X, T)$ is collectionwise normal. 
Proof. Let $F$ be a discrete collection of subsets of $X$. For each $x \in \cup F$ we let $C_{x}$ denote the unique set in $F$ which contains $x$. Thus $C_{x} \cap C_{y} \neq \varnothing$ iff $C_{x}=C_{y}$. Since $F$ is discrete, for each $x \in \cup F$ there exists $V_{x} \in B_{x}$ such that

$$
V_{x} \cap(\cup F) \subset C_{x},
$$

and since $B$ is cohesive, for each $x \in \cup F$ we can choose $U_{x} \in B_{x}$ such that (*) $z \in U_{x}$ implies that $x \in V_{z}^{x} \subset V_{x}$ for some $V_{z}^{x} \in B_{z}$.

We now let $O_{x}=\cup\left\{\operatorname{Int}\left(U_{p}\right): p \in C_{x}\right\}$ for all $x \in \cup F$. Since each $p$ determines a unique set $U_{p}$,

$$
O_{x}=O_{y} \text { if } C_{x}=C_{y} .
$$

Clearly $C_{x} \subset O_{x}$ and each $O_{x}$ is open. Moreover, $O_{x} \cap(\cup F)=C_{x}$ by (1), since $U_{p} \subset V_{p}$. So to prove that $X$ is collectionwise normal, we have yet to show that the members of $\left\{O_{x}: x \in \cup F\right\}$ are pairwise disjoint.

To this end, we suppose that $O_{x} \cap O_{y} \neq \varnothing$ for some $x, y$ and we show that $O_{x}=O_{y}$. The definition of $O_{x}$ and $O_{y}$ yields $p \in C_{x}$ and $q \in C_{y}$ such that $\operatorname{Int}\left(U_{p}\right) \subset O_{x}, \operatorname{Int}\left(U_{q}\right) \subset O_{y}$, and $\operatorname{Int}\left(U_{p}\right) \cap \operatorname{Int}\left(U_{q}\right) \neq \varnothing$. Thus there exists $z \in U_{p}$ $\cap U_{q} \cap D$, since $D$ is dense in $X$. Then by (*), $z \in U_{p}$ implies there exists $V_{z}^{p} \in B_{z}$ such that $p \in V_{z}^{p} \subset V_{p}$, and $z \in U_{q}$ implies there exists $V_{z}^{q} \in B_{z}$ such that $q \in V_{z}^{q} \subset V_{q}$.

But $z \in D$ so that $B_{z}$ is monotone, and we may assume w.l.o.g that $V_{z}^{q} \subset V_{z}^{p}$. By the above we therefore have $p, q \in V_{p}$. Since $p \in C_{x}$ and $q \in C_{y}, V_{p} \cap C_{x} \neq \varnothing$ and $V_{p} \cap C_{y} \neq \varnothing$. But $V_{p} \cap(\cup F) \subset C_{p}$ (see (1)), so $C_{p} \cap C_{x} \neq \varnothing$ and $C_{p} \cap C_{y}$ $\neq \varnothing$. Thus $C_{x}=C_{p}=C_{y}$, and (2) implies $O_{x}=O_{y}$.

Since the properties of a neighborhood basis $B_{p}$ being monotone and the family $\left\{B_{p}: p \in X\right\}$ being cohesive are clearly hereditary, we can say

COROLlARY 2.7. A $T_{1}$ space $X$ which has a monotone neighborhood basis $B_{p}$ at each point $p$ such that $\left\{B_{p}: p \in X\right\}$ is cohesive is hereditarily collectionwise normal (or equivalently, completely collectionwise normal).

Corollary 2.7 and a result by McAuley [4] imply

Corollary 2.8. Any semimetric space $(X, d)$ in which the family $\left\{B_{p}: p \in X\right\}$ with $B_{p}=\{S(p, 1 / n): n \in N\}$ is cohesive is completely collectionwise normal and paracompact.

On the other hand, a well-known example of McAuley (see [5 or 6, p. 175]) demonstrates that a semimetric space may be paracompact and even hereditarily separable although the neighborhoods $S(p, 1 / n)$ do not generate a cohesive family. That the $1 / n$ neighborhoods do not generate a cohesive family follows from Theorem 2.5 and the fact that McAuley's space is separable but not second countable. Now McAuley's space is not developable. Another well-known example, the "tangent disc" toplogy [6, p. 176] is a regular developable space which is not normal and therefore, by Theorem 2.6, the family $\left\{B_{p}: p \in X\right\}$ with $B_{p}=$ $\left\{\operatorname{St}\left(p, G_{n}\right): n \in N\right\}$ is not cohesive $\left(\operatorname{St}\left(p, G_{n}\right)=\cup\left\{0 \in G_{n}: p \in 0\right\}\right)$. However, if we combine the cohesive property with developability, we obtain metrizability. 
Corollary 2.9. $A T_{1}$ space $X$ is metrizable iff there is a refined development $\left\{G_{n}\right.$ : $n \in N\}$ of $X$ such that $\left\{B_{p}: p \in X\right\}$ with $B_{p}=\left\{\operatorname{St}\left(p, G_{n}\right): n \in N\right\}$ is cohesive.

Proof. The "necessity" is immediate. To verify " sufficiency" note that since $G_{n+1}$ refines $G_{n}$, each $B_{p}$ is monotone, so that $X$ is collectionwise normal. But as is well known, any collectionwise normal developable space is metrizable.

3. Metrizability criteria. A theorem in [3] states

THEOREM [3]. $A T_{3}$ space $(X, T)$ is metrizable iff there is a semimetric d compatible with $T$ such that

(i) $\lim _{n} d\left(x_{n}, y_{n}\right)=\lim _{n} d\left(x_{n}, p\right)=0$ implies $\lim _{n} d\left(y_{n}, p\right)=0$.

The $T_{3}$ requirement in the above result is redundant. For suppose that $(X, T)$ is a $T_{1}$ space and $d$ is a semimetric compatible with $T$ for which (i) above holds. Thus $S(p, 1 / n)$ is indeed a neighborhood of $p$, and it is a simple matter to show that for any $p \in X$ and $n \in N$, there exists $k=K(p, n) \geqslant n$ such that $p \in S(x, 1 / k) \subset$ $S(p, 1 / n)$ for all $x \in S(p, 1 / k)$ (remember, $x \in S(p, 1 / k)$ iff $p \in S(x, 1 / k)$ since $d$ is "symmetric"). Thus if $B_{p}=\{S(p, 1 / n): n \in N\}$, then $\left\{B_{p}: p \in X\right\}$ is cohesive-in a uniform way. Consequently, Theorem 2.6 applies, so that $(X, T)$ is certainly $T_{3}$ and we have

Proposition 3.1. A $T_{1}$ space $(X, T)$ is metrizable iff there is a semimetric d compatible with $T$ such that

(i) $\lim _{n} d\left(x_{n}, p\right)=\lim _{n} d\left(x_{n}, y_{n}\right)=0$ implies $\lim d\left(y_{n}, p\right)=0$.

Now a $T_{1}$ space $(X, T)$ which has a continuous semimetric compatible with $T$ is developable but need not even be normal [2]. The next result, which we believe (surprisingly) to be new, tells us what to add to continuity to obtain metrizability.

TheOREM 3.2. A $T_{1}$ space $(X, T)$ is metrizable iff there is a semimetric $d$ compatible with $T$ which is uniformly continuous.

$A$ semimetric $d$ is continuous at $(a, b)$ iff for any $\varepsilon>0$ there is $a \delta>0$ such that (ii) $|d(x, y)-d(a, b)|<\varepsilon$ if $\max \{d(x, a), d(y, b)\}<\delta$. Of course, $d$ is uniformly continuous if $\exists \delta>0$ such that (ii) holds for any $(a, b)$ and $(x, y)$ in $X \times X$.

Proof (OF Theorem 3.2). If $(X, T)$ is metrizable and $d$ is a metric compatible with $T$, the triangle inequality yields

$$
|d(x, y)-d(a, b)| \leqslant d(x, a)+d(y, b) \leqslant 2 \max \{d(x, a), d(y, b)\}
$$

so that (ii) above is satisfied for any $(x, y)$ and $(a, b)$ and any $\varepsilon$ if $\delta=\varepsilon / 2$; thus $d$ is uniformly continuous.

Conversely, suppose that $d$ is uniformly continuous and that $d\left(x_{n}, p\right) \rightarrow 0$ and $d\left(x_{n}, y_{n}\right) \rightarrow 0$, and let $\varepsilon>0$. By uniform continuity $\exists \delta>0$ such that $d\left(p, y_{n}\right)=\left|d\left(y_{n}, p\right)-d\left(x_{n}, x_{n}\right)\right|<\varepsilon$ if (iii) $\max \left\{d\left(y_{n}, x_{n}\right), d\left(p, x_{n}\right)\right\}<\delta$. Since $d\left(x_{n}, y_{n}\right) \rightarrow 0$ and $d\left(x_{n}, p\right) \rightarrow 0$, there exists $k$ such that (iii) holds for $n \geqslant k$; i.e., $d\left(y_{n}, p\right)<\varepsilon$ if $n \geqslant k$. Thus $\lim d\left(y_{n}, p\right)=0$, and $(X, T)$ is metrizable by Proposition 3.1. 
(For a study of continuity and uniform continuity in the context of generalized metric spaces see [1].)

In the comments preceding Proposition 3.1 we observed that the requirement (i) induced a local uniform cohesiveness on the $1 / n$ neighborhoods, a concept we now utilize.

THEOREM 3.3. $A T_{1}$ space $(X, T)$ is metrizable iff there is a countable monotone local base $\left\{V_{n}(p): n \in N\right\}$ at each $p \in X$ such that $(* *)$ for each $n \in N, \exists k=$ $k(n, p) \geqslant n$ such that $p \in V_{k}(x) \subset V_{n}(p)$ for all $x \in V_{k}(p)$.

Proof. The "necessity" follows immediately with $V_{n}(p)=S(p, 1 / n)$. To prove that the condition is sufficient, for each $n \in N$ we let $G_{n}=\left\{V_{k(n, x)}(x): x \in X\right\}$ where $k=k(n, x)$ is chosen to satisfy (**). We assert that $G=\left\{G_{n}: n \in N\right\}$ is a development. Clearly, each $G_{n}$ is an open cover of $X$, so we have yet to show that $\left\{\operatorname{St}\left(p, G_{n}\right): n \in N\right\}$ is a local base at $p$ for $p \in X$. To see this, let $p \in 0$ for some $0 \in T$. Since $B_{p}$ is a local base at $p$, we can choose $n$ such that $V_{n}(p) \subset 0$. We prove that $\operatorname{St}\left(p, G_{k(n, p)}\right) \subset V_{n}(p)$. Note that

$$
\operatorname{St}\left(p, G_{k(n, p)}\right)=\bigcup\left\{V_{k(k(n, p), x)}(x): p \in V_{k(k(n, p), x)}(x)\right\} \text {. }
$$

If $p \in V_{k(k(n, p), x)}(x)$, the definition of $k(k(n, p), x)$ and $(* *)$ imply $x \in$ $V_{k(k(n, p), x)}(p) \subset V_{k(n, p)}(x)$. But

$$
k(k(n, p), x) \geqslant k(n, p) .
$$

Thus by monotonicity $x \in V_{k(n, p)}(p)$, so the designation of $k(n, p)$ and (**) imply $p \in V_{k(n, p)}(x) \subset V_{n}(p)$. Thus (2) yields $V_{k(k(n, p), x)}(x) \subset V_{n}(p)$, and by (1) we have $\operatorname{St}\left(p, G_{k(n, p)}\right) \subset V_{n}(p)$ as desired; i.e., $(X, T)$ is developable. But by Theorem 2.6 $(X, T)$ is also collectionwise normal and therefore metrizable, since any collectionwise normal developable space is metrizable.

In closing we note that since Urysohn's Theorem tells us that any regular second countable $T_{1}$ space is metrizable, we can apply Theorems 2.5 and 2.6 to conclude

Corollary 3.4. A separable $T_{1}$ space $X$ is metrizable iff there is a countable monotone local base $B_{p}$ at each $p \in X$ such that $\left\{B_{p}: p \in X\right\}$ is cohesive.

\section{REFERENCES}

1. F. Cagliari and M. Cicchese, Continuity conditions and convergence properties in generalized metric spaces, Riv. Mat. Univ. Parma (4) 2 (1976), 329-336.

2. H. Cook, Cartesian products and continuous semimetrics, Proceedings of Point Set Topology Conference (Editor, E. Grace), Arizona State Univ., Tempe, 1967.

3. R. W. Heath, On certain first countable spaces, Topology Seminar, Wisconsin Univ. Press and Princeton Univ. Press, Princeton, N. J., 1966, pp. 103-113.

4. L. F. McAuley, A note on complete collectionwise normality and paracompactness, Proc. Amer. Math. Soc. 9 (1958), 796-799.

5.,$A$ relation between perfect separability, completeness, and normality in semimetric spaces, Pacific J. Math. 6 (1956), 315-326.

6. J. Seebach, Jr. and L. A. Steen, Counterexamples in topology, 2nd ed., Springer-Verlag, Berlin and New York, 1978.

Department of Mathematics, Bradley University, Peoria, Illinois 61625 